\title{
FIRST RESULTS OF THE BORDEAUX MERIDIAN CIRCLE EQUIPPED WITH A CCD DETECTOR
}

\author{
Y.RÉQUIÈME, G.MONTIGNAC, J.F.LE CAMPION, F.BOSQ and F.CHAUVET \\ Bordeaux Observatory, B.P.89, F-33270 Floirac, France \\ and \\ P.BENEVIDES-SOARES and R.TEIXEIRA \\ Instituto Astronomico e Geofisico USP, 04301-904 São Paulo, Brazil
}

The Bordeaux automatic meridian circle has been working for 10 years with a tracking photoelectric micrometer. This micrometer was recently replaced by a focal CCD camera in order to extend the differential position measurements to much fainter stars with improved accuracy. The main features of the Bordeaux CCD instrument are presently :

$-20 \mathrm{~cm}(\mathrm{f} / 12)$ meridian refractor : $87.5 \mathrm{H} / \mathrm{mm}$.

- Thomson 7895A thick CCD (MPP technology) 512*512, cooled at -40 deg.C. by

Peltier thermoelectric elements and water circulation. Pixel size : 19 microns (1.65").

- Filter GG495 + BG38 : effective bandwidth 5200-6800 A.

- Imaging in drift-scanning mode. Equivalent exposure time $56 \sec \delta$ seconds.

- Real-time continuous display of the observed star field.

Preliminary tests on the sky carried out from March 1994 onwards give clear evidence of slow image motion due the atmosphere (Fig.1 : raw differences night to night).

Observing narrow strips with the instrument stationary and taking as reference the final Hipparcos and Tycho positions, the expected accuracy on the positions is 0.03 "- 0.06 " for four-fold measurements in the magnitude range $9<\mathrm{V}<15$ (Fig.2). In addition, magnitudes will be obtained with a mean error of $0.1-0.3 \mathrm{~m}$.
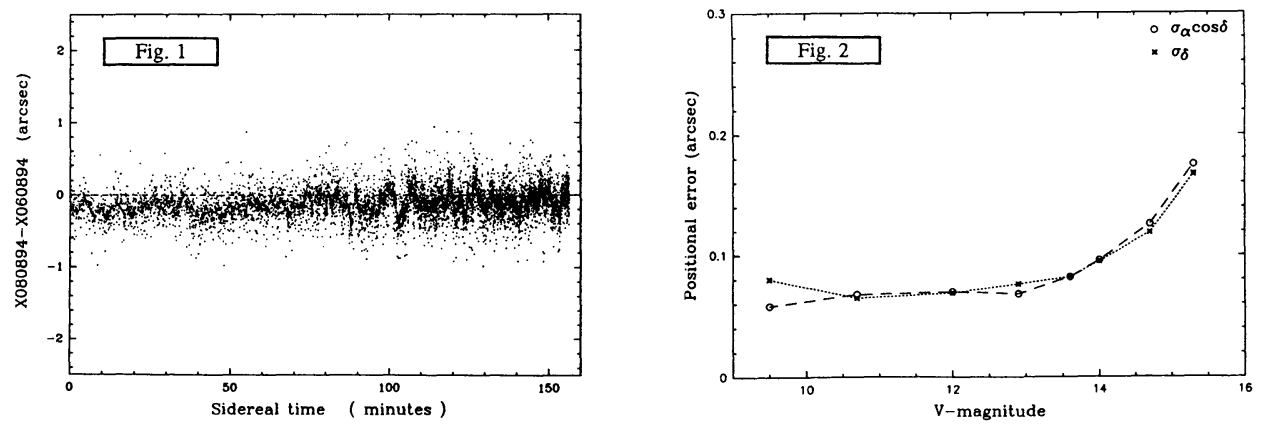

Scientific programmes are extension of the Hipparcos-Tycho frame up to $V=15-16$, link to the VLBI primary reference frame via faint radiostars, positions of satellites of planets and asteroids, survey of faint star proper motions in selected areas.

The first camera will be moved soon on the São Paulo meridian circle, a new one with a $1024 * 1024$ CCD will be mounted on the Bordeaux meridian instrument.

ACKNOWLEDGEMENTS - This project was partially supported by the French CNRS (INSU) and the Aquitaine Region, and also by CNPq (Brazil) and CNRS through travel grants. 